\title{
Le sujet lecteur dans la classe : éléments pour un état des lieux des pratiques dans le secondaire
}

\section{Magali Brunel}

\section{OpenEdition}

1 Journals

Édition électronique

URL : http://journals.openedition.org/recherchestravaux/655

DOI : 10.4000/recherchestravaux.655

ISSN : 1969-6434

Éditeur

UGA Éditions/Université Grenoble Alpes

\section{Édition imprimée}

Date de publication : 31 décembre 2013

Pagination : 117-130

ISBN : 978-2-84310-267-7

ISSN : 0151-1874

\section{Référence électronique}

Magali Brunel, « Le sujet lecteur dans la classe : éléments pour un état des lieux des pratiques dans le secondaire », Recherches \& Travaux [En ligne], 83 | 2013, mis en ligne le 01 juillet 2015, consulté le 08 septembre 2020. URL : http://journals.openedition.org/recherchestravaux/655; DOI : https://doi.org/ 10.4000/recherchestravaux.655 


\section{Le sujet lecteur dans la classe : éléments pour un état des lieux des pratiques dans le secondaire}

Tandis que de nombreux travaux ont pu conduire à une théorisation de plus en plus précise du concept de sujet lecteur et que plusieurs articles ont abordé sa place dans les programmes ${ }^{\mathrm{I}}$, nous nous proposons de porter un regard sur la réalité des pratiques de classe, des réalisations pédagogiques effectives, non en focalisant sur une expérimentation spécifique, ou une pratique innovante, mais en nous donnant pour objectif d'évaluer en quels termes, aujourd'hui, les enseignants réfléchissent à l'enseignement de la lecture. Nous analyserons en particulier la place qu'ils accordent à la pratique de la lecture subjective et aux différents types d'activités didactiques qu'ils adoptent pour enseigner une posture engageant un sujet lecteur. Il s'agit donc de présenter une forme d'état des lieux des pratiques actuelles, de leur variété et des difficultés qui demeurent.

Rappelons, en guise de point de comparaison, quelques éléments des conclusions auxquelles aboutissait, il y a près de dix ans, le rapport de l'IGEN sur les pratiques de lecture des enseignants du secondaire. Le rapporteur regrettait le fait que bien souvent les textes littéraires étudiés "deviennent le lieu de passage» - de notions et d'outils - et non de "rencontre» avec des textes littéraires, et présentait une séance type de lecture, reflétant la majorité des pratiques :

I. Nous pensons à l'article de M. Lebrun, «L'émergence et le choc des subjectivités de lecteurs de la maternelle au lycée grâce au débat interprétatif ouvert par les comités de lecture», Le Sujet lecteur, lecture subjective et enseignement de la littérature, 2004, ou à celui de G. Langlade, "Sortir du formalisme, accueillir les lecteurs réels», Le Français aujourd'hui, 2004/2, p. 85-96. 
Sans que soient réellement accueillies, traitées et utilisées les réactions des lecteurs, [le professeur] fait surgir trois axes d'étude qui vont structurer le commentaire composé oral qui tient lieu désormais de «lecture analytique ${ }^{2}$.

Il encourageait alors les enseignants à diversifier leurs pratiques et leurs approches en s'appuyant notamment sur les ressources des lectures cursives. En un mot, il encourageait une véritable rencontre entre des œuvres qui ne soient pas des prétextes, et des élèves qui soient pris en compte dans leur individualité. C'est donc notamment en regard avec ce constat que l'on se propose de dresser un nouvel état des lieux des pratiques de lecture, et, en particulier, de la place que peut occuper l'enseignement de la lecture subjective en classes de collège et lycée.

\section{Présentation des données recueillies}

Nous avons, pour avancer dans ce questionnement, utilisé un recueil de données spécifiques : il s'agit de plus de cent dossiers, présentés par des enseignants du secondaire, candidats à l'obtention du CAPES interne. Ceux-ci constituent en effet, depuis la session 20I2, un dossier faisant état de leurs compétences professionnelles et présentant une exploitation didactique et pédagogique récente dont ils proposent ensuite une analyse ${ }^{3}$.

Bien entendu, les documents recueillis doivent être interrogés avec précaution : ils ne sont pas a priori l'image de toutes les pratiques. Leurs auteurs présentent un statut de vacataires, n’ont pas bénéficié de formation initiale; cependant, bien souvent, ils enseignent dans les établissements depuis plus de trois ans, ont pu bénéficier de formations voire d'inspections ${ }^{4}$ et de stages d'accompagnement pour la préparation à l'épreuve. De plus, ils appartiennent à des équipes pédagogiques, apprennent souvent par modélisation, et échangent sur leur pratique, surtout sans doute au moment de soumettre un tel dossier à l'examen. Enfin, bien entendu, de nombreux enseignants titulaires sont issus de ces mêmes concours internes, et, réciproquement, un certain nombre d'entre eux est titularisé à l'issue de l'examen. Pour ces différentes raisons, il nous semble que les données analysées peuvent présenter un panel intéressant et assez proche

2. Extrait du rapport La Mise en œuvre du programme de français en classe de seconde, oct. 2003, p. 18.

3. "Le candidat développe plus particulièrement, à partir d'une analyse précise et parmi ses réalisations pédagogiques dans la discipline concernée par le concours, celle qui lui paraît la plus significative, relative à une situation d'apprentissage et à la conduite d'une classe qu'il a eue en responsabilité », arrêté du 27 avril 20II, complété par sa note de commentaire.

4. De nombreux rapports d'inspection ont en effet été joints aux dossiers. 
des pratiques enseignantes dans notre discipline. En outre, le corpus offre plusieurs intérêts pour l'analyse: d'une part, les propositions didactiques recueillies sont toutes récentes et ont été sélectionnées par les enseignants comme étant les plus «significatives» de leur activité professionnelle; elles constituent donc, pour eux, des expériences qu'ils considèrent être les plus favorables pour être jugés. D'autre part, le nombre important de dossiers analysés et leur provenance de tout le territoire national constituent des gages de fiabilité.

Nos données se composent donc de cent deux dossiers, dont quatre-vingtdix-huit présentent effectivement une unité didactique et pédagogique d'enseignement dans la discipline du français : activité souvent centrée sur des travaux de lecture. Notre analyse a permis de mettre en évidence trois constats que nous nous proposons de développer :

- la réalité d'une réflexion des enseignants sur les pratiques scolaires de lecture, sur leur lien ou leur distance avec les pratiques personnelles des élèves;

- la diversité de pratiques offrant la possibilité à la subjectivité du lecteur de s'exprimer;

- la persistance de difficultés sur le développement d'un enseignement de la lecture subjective.

\section{Un véritable questionnement sur les pratiques de lecture}

Nous pouvons, d'une part, relever le fait qu'une large partie des candidats se questionne sur l'activité même de lecture : nombre d'entre eux soulignent leur difficulté à concilier les compétences réelles de lecture des élèves avec les exigences du programme officiel et insistent sur la difficulté de certains textes et le manque de motivation des élèves. Ils mettent alors en évidence la tension entre plusieurs exigences de leur profession : d'un côté préparer les élèves à des évaluations, des examens, de l'autre, chercher à les motiver dans leur lecture, à les rendre plus autonomes. Telle est la préoccupation de cette enseignante de classe de première, qui s'interroge ainsi :

Comment concilier les épreuves du baccalauréat, apporter les outils d'une culture générale et proposer aux élèves une vraie réflexion sur le monde qui les entoure, sur la société dans laquelle ils vivent? Et surtout, dans les conditions d'une année d'examen, comment susciter l'envie? (Dossier n $\left.{ }^{\circ} 25\right)$

D'autres interrogations surgissent au sujet de la notion de goût, déjà largement étudiée notamment par Jean-François Massols, dès I999 : si la

5. Nous renvoyons à l'ouvrage Lecture privée et lecture scolaire, coordonné par P. Demougin et J.-F. Massol, CRDP Grenoble, I999. 
manifestation du goût des élèves est envisagée par les textes officiels, et l'objectif de "donner le goût» souligné, les enseignants sont conscients de l'écart entre lecture spontanée privée des élèves et lecture scolaire. En même temps, ils se disent convaincus de la nécessité de stimuler le goût de lire dans leur enseignement. Cependant, si cette difficulté est soulevée, elle est souvent rapidement dissimulée sous des propositions convenues. Ainsi, cet enseignant reprend, dans sa conclusion, la question du goût de lire, qu'il avait abordée comme problème en introduction :

J'espère avoir non seulement bien préparé les élèves aux épreuves de français, mais aussi avoir développé ou fait naître chez eux une conscience esthétique et un goût, sinon pour la littérature, du moins pour la lecture. (Dossier n $\left.{ }^{\circ} 74\right)$

Certains dossiers dépassent cependant la seule évocation pour s'engager sur un questionnement de leur pratique coutumière d'enseignement de la lecture : ainsi, plusieurs enseignants présentent en début de dossier le constat des limites de certains dispositifs, comme celui du questionnaire, ou celui de la lecture analytique telle qu'ils la pratiquent régulièrement. Il en est ainsi dans ce dossier où l'enseignante justifie son choix pour la séquence qu'elle expose :

En règle générale, les élèves ont pour chacune de leur lecture analytique un questionnaire avec au moins quatre questions. [...] J'ai changé de méthode pour ce dernier objet d'étude car les élèves restent enfermés dans ce questionnement, ils ne cherchent pas à expliquer le texte mais juste à remplir des «cases». (Dossier $\left.\mathrm{n}^{\circ} 25\right)$

Constatant l'artificialité du dispositif de lecture qu'elle emploie habituellement, elle cherche ainsi à faire évoluer ses pratiques de lecture.

Enfin, certains enseignants cherchent à diversifier les postures de lecteurs qu'ils conduisent à adopter : nous citerons pour exemple quelques extraits d'un dossier où la candidate explique les raisons qui l'ont poussée à travailler en classe Vendredi ou la vie sauvage de Michel Tournier :

L'œuvre me semblait thématiquement attrayante. Les récits d'aventure ont souvent la faveur du public adolescent [...] le plaisir du texte est lié, pour les élèves, à la possibilité qu'ils ont de s'identifier aux personnages en les percevant plus comme personnes que comme êtres de papiers. (Dossier $\mathrm{n}^{\circ}$ 9I)

L'enseignante envisage dans ce cas de favoriser une posture de compréhension-identification, proche de la posture de lecture du texte-action que décrit Dominique Bucheton. Le livre est alors le miroir de son lecteur, il est le «lu», selon Michel Picard ${ }^{6}$. Le professeur poursuit en une nouvelle perspective :

6. M. Picard, La Lecture comme jeu, Paris, Minuit, 1986. 
Mon rôle est de les amener à s'interroger sur le sens, ou les sens, de la fable au-delà de l'histoire proprement dite, car, à travers la métaphore de l'aventure, l'auteur nous parle des difficultés de la vie réelle et des moyens que nous avons d'y faire face. (Dossier n ${ }^{\circ}$ 9I)

Elle considère en outre que, par son étayage, elle pourra faire émerger une autre posture, par laquelle le texte interroge le lecteur sur son propre rapport au monde - c'est la lecture reflet de Bucheton - ou encore permettre d'interpréter le système de valeurs qu'il met en scène - ce que Dominique Bucheton présente comme « un passage à la perception du texte comme métaphore [qui] permet l'ouverture de nouvelles significations ${ }^{7}$ ». L'enseignant sollicite alors, de manière productive, une même œuvre pour concevoir des activités de lecture engageant des postures différentes, activant ainsi diverses compétences de lecteur.

Ainsi, de nombreux enseignants font état de tensions entre les contraintes institutionnelles, leurs pratiques conventionnelles et leurs propres visions de la lecture. Ceci les conduit à développer des réflexions sur le goût de la lecture, et à envisager des propositions didactiques, qu'ils concentrent notamment dans la première séance, pour développer la motivation des élèves ${ }^{8}$. Ils cherchent ainsi à concilier, dans leur enseignement, plaisir et apprentissage, postures d'engagement personnel et de mise à distance.

\section{Une variété de pratiques sollicitant une posture de sujet lecteur}

Dans un deuxième temps, nous nous proposons d'analyser, dans les dossiers, les descriptions de pratiques de lecture mettant en jeu une posture de lecture subjective. Tout d'abord, un commentaire doit être porté sur leur nombre : sur les 98 dossiers présentant une exploitation de textes littéraires, 22 prévoient des activités de lecture subjective', soit plus de $20 \%$. Parmi eux, les deux tiers proposent plus de deux activités de ce type, et prévoient donc de laisser une réelle place, au sein de la séquence, à l'émergence du sujet lecteur. Il nous semble

7. Dans ce cas, l'enseignante ne vise pas à faire exercer la posture «lettrée» du texte-objet, où l'œuvre est analysée dans ses stratégies formelles, ses mécanismes de fonctionnement, ses effets, p. I45.

8. Différentes stratégies sont mises en place pour impliquer personnellement l'élève dans la séquence et dans sa lecture : discussion libre, mise en commun des représentations sur un thème, un genre ou un auteur, lecture de l'image, censée plus facilement déclencher une motivation, ou activités sur l'objet-livre, censées favoriser un rapport tantôt "personnel », "concret» ou "ludique», à l'acte de lecture.

9. Si l'on s'en tient aux définitions développées notamment dans B. Louichon, A. Rouxel (dir.), Du Corpus scolaire à la bibliothèque intérieure, Rennes, Presses universitaires de Rennes, coll. «Païdeia», 2010. 
que ces chiffres manifestent une évolution par rapport au constat qu'établissait le rapporteur de l'inspection générale en 2003. Nous nous proposons donc d'identifier précisément ces différentes propositions didactiques, que nous avons classées en trois ensembles.

\section{Les activités de lecture subjective se réalisant dans des supports écrits}

Ces activités invitent les élèves à garder une trace de leurs lectures et rendent visible une activité de lecture que les activités scolaires traditionnelles ne permettent pas de saisir, de façon à développer l'adoption d'une posture de lecture subjective. En effet, comme le souligne Patrick Demougin «si enseigner la littérature consiste pour une bonne part à aider le sujet lecteur à se construire, il faut l'aider à construire cette parole intérieure, à jouer et rejouer les moments de sa construction ${ }^{\mathrm{I0}}$ ». Par ces "traces écrites» de lecture, un aspect intime de la construction du lecteur est alors exercé. Il s'agit donc de s'appuyer sur l'écrit pour développer les qualités d'appropriation et d'interprétation des élèves. Ainsi, l'appropriation, qui passe souvent par l'identification, centrale dans la construction d'un sujet lecteur, est particulièrement sollicitée dans certaines propositions, comme la constitution de journaux et carnets de lecteur dans lesquels les élèves évoquent leurs impressions, témoignent de leur "lecture affective», de leur «itinéraire personnel de lecteur» (dossier $\left.n^{\circ} 73\right)$. Les enseignants exploitent également des situations écrites collectives qui facilitent la compréhension et l'interprétation. L'un d'entre eux, après avoir insisté sur le fait que la narration des Contes de la bécasse est riche et complexe, et s'apparente, dans les récits proposés par les élèves à "la construction d'une carte, d'une topographie», envisage également de faire réaliser une carte heuristique :

Je réfléchis aujourd'hui à la manière dont j'aurais pu matérialiser cette pratique par une trace écrite. La piste de la réalisation pédagogique d'une carte heuristique à l'aide d'un logiciel de carte mentale m'intéresse dans la mesure où elle me permettrait de créer un document qui peut être complété au fur et à mesure et remis aux élèves à la fin du parcours! (Dossier n ${ }^{\circ}$ 19)

Le deuxième intérêt de telles activités est le développement de qualités réflexives, puisque, ainsi que le rappelle Jean-Louis Dufays, les compétences

IO. De nombreuses références étant faites au cours de cet article au volume collectif de A. Rouxel, G. Langlade (dir.), Le sujet lecteur, lecture subjective et enseignement littéraire, Rennes, Presses universitaire de Rennes, 2004, il sera désormais mentionné LSL. P. Demougin, «Le lecteur et sa parole : traces écrites d'une parole recomposée dans l'acte de lecture", LSL, 2004, p. II7-I27. 
métacognitives «différencient le plus nettement les lectures experts des lecteurs faibles ${ }^{\mathrm{II}}$. Permettre alors à l'élève d'analyser sa lecture, d'exprimer son goût et de justifier ses choix favorise l'acquisition d'une métacognition. Trois dossiers s'engagent dans cet objectif : ainsi, une enseignante propose de composer, dans le carnet de lecture, une première page dans laquelle l'élève peut noter tout ce que lui évoque le titre Alice au pays des merveilles, puis, au fil de la lecture, elle propose de prendre des notes et de les comparer à ses représentations de la première page. Par la confrontation de ces deux approches de l'œuvre, l'élève est ainsi invité à adopter une posture réflexive.

Ajoutons le fait qu'écrire sur sa lecture permet également de développer une identité de lecteur ${ }^{\mathrm{r} 2}$. En effet, les textes dans lesquels les élèves se sont investis, ceux qu'ils aiment, sont aussi ceux qui les représentent, qui les construisent. Dès lors, favoriser l'émergence ou la prise de conscience d'une identité qui s'élabore de manière très progressive, permet aux élèves de prendre place dans une communauté culturelle, de s'y intégrer. C'est notamment l'intérêt de l'atelier d'écriture proposé par une enseignante en sixième : elle présente une illustration du Petit Chaperon rouge de Perrault par Sarah Moon, et amène les élèves à écrire à partir de la phrase inductrice suivante : " je me souviens de la première fois que... $\left(\right.$ dossier $\left.\mathrm{n}^{\circ} 56\right)$. S'engageant ainsi dans la reconstitution d'une mémoire de lecteur, centrée sur une œuvre que tous les enfants ont écoutée, lue ou regardée sous la forme d'album, le professeur permet aux élèves de prendre conscience de l'existence de cette mémoire, et peut-être du rôle qu'elle peut jouer dans leur lecture présente du conte.

Enfin, les traces écrites des lectures conduisent également les élèves à développer le plaisir de l'écriture. Bien souvent, la dimension esthétique des travaux présentés est en effet soulignée par les enseignants, par exemple dans ce dossier dans lequel le professeur explique : «Cette agréable séance a illustré le plaisir que les élèves avaient pu prendre à la lecture mais aussi à la réalisation de leur journal.» (dossier $\mathrm{n}^{\circ}$ 73) L'ensemble de ces activités permet aux élèves de faire résonner les œuvres lues dans leur propre écrit. Comme le souligne Violaine Houdart Mérot, «transformer sa lecture en écriture pourrait être l'une des manières de rendre la lecture véritablement créatrice et le lecteur acteur à part entière ${ }^{\mathrm{I}_{3}}$ ».

Ainsi, plus de dix propositions d'activité de lecture subjective réalisées sur des supports écrits sont présentées et témoignent de la vitalité de ce type de pratiques.

II. S. de Croix et J.-L. Dufays, «Se raconter pour mieux se percevoir comme sujet lecteur», LSL, 2004, p. 153-165, p. I54.

I2. Comme l'explique A. Rouxel dans «Autobiographie de lecteur et identité littéraire», $L S L$, p. I37-I5I.

13. V. Houdard-Mérot, "De la critique d'admiration à la lecture scriptible», LSL, p. 223-232, p. 23 I. 


\section{Les activités de lecture subjective se réalisant dans des activités orales}

Près de dix dossiers incluent des pratiques de lecture subjective prenant appui sur des activités orales, qui permettent, elles aussi, de développer certaines compétences favorisant une lecture subjective. Ainsi tout d'abord, les enseignants encouragent des pratiques orales car elles facilitent les réactions spontanées des élèves, leur capacité à dire quelque chose sur un texte, à exprimer ce qu'il leur $\mathrm{dit}^{\mathrm{I}}$. Ces réactions, qui consistent souvent en un jugement moral sur les actions des personnages, constituent le signe d'une implication dans la lecture, et leur développement, par le biais de l'échange, permet d'accentuer cette posture. Comme le souligne Gérard Langlade : «dans cette distance participative faite d'aperçus psychologiques, de jugements moraux, de séduction ou de répulsion, etc., se lisent et se lient l'œuvre et le sujet lecteur ${ }^{15}{ }^{\prime}$. Tel est l'objectif de cette enseignante de première, envisageant l'étude de Gargantua, qui légitime sa volonté d'encourager les réactions subjectives de ses élèves en se référant à Rabelais lui-même : «il y a sous l'écorce tant de belles significations pour l'instruction de tous que chaque lecteur, selon ses affections, en tirera lui-même des sens qui lui seront particuliers ${ }^{16}{ }^{\prime}\left(\right.$ dossier $\left.n^{\circ}{ }^{16}\right)$.

Le deuxième intérêt d'activités orales consiste à permettre, par la confrontation des lectures particulières des élèves, de mettre à jour les ambiguïtés, les indéterminations du texte qui se révèle ainsi résistant, ambigu. Au-delà, ces activités amènent également les élèves à percevoir la spécificité de leur lecture, voire parfois, ses errances, ses erreurs. Comme le souligne Vincent Jouve, le processus interprétatif se fondant sur les «lieux d'incertitude» du texte, la confrontation des lectures subjectives recèle un véritable intérêt pédagogique :

C'est finalement lorsque les configurations subjectives du lecteur sont remises en cause par le texte (c'est-à-dire lorsque la subjectivité est accidentelle) que l'expérience de retour sur soi est la plus frappante. Le lecteur est alors amené à réfléchir sur ce qui l'a conduit à projeter dans le texte ce qui n'y était pas ${ }^{17}$.

Ainsi, dans un des dossiers présentés, le professeur met en évidence les divergences d'interprétation d'un épisode du Petit Prince :

La fin du texte se révèle aussi problématique pour certains élèves. En effet, même après avoir relevé les expressions qui mettent en évidence une séparation, un adieu, les élèves n’ont pas la même interprétation de la fin du récit : certains élèves

I4. P. Demougin, LSL, p. II7-I27.

I5. G. Langlade, «Le sujet lecteur auteur de la singularité de l'œuvre», LSL, p. 8I-9I.

I6. Citation présentée par l'enseignante pour justifier le choix de l'œuvre étudiée.

I7. V. Jouve, «La lecture comme retour sur soi : de l'intérêt pédagogique des lectures subjectives", LSL, p. I05-II5, p. I05. 
comprennent que le petit prince est mort et d'autres ne peuvent envisager cette possibilité et estiment que le petit prince est retourné sur sa planète. (Dossier $n^{\circ} 87$ )

Cet exemple est particulièrement frappant et révèle combien l'échange des lectures particulières peut être intéressant. Il montre que la lecture joue sur l'identité même du lecteur qui peut en être nourri voire fragilisé : ainsi les différentes interprétations présentées par le professeur ne portent-elles pas le signe des différents rapports à la mort des élèves et le fait de les mettre en question ne les ébranle-t-il pas profondément?

Un autre ensemble d'activités orales engage une mise en voix ou une mise en scène qui peut permettre "d'incorporer le texte à soi", comme l'exprime si joliment Annie Rouxel. Ainsi, un professeur explique qu'il propose «des pauses exceptionnelles", consacrées à faire raconter à des élèves volontaires une nouvelle de Maupassant qu'ils ont lue :

C'est une situation complexe pour l'élève. Elle requiert tout son engagement et met en jeu ses désirs et ses affects dans une situation d'apprentissage. [...] malgré ma difficulté à gérer parfois la reprise du cours, ces situations ont souvent donné lieu à de véritables échanges authentiques où les élèves, libérés de la parole du professeur, écoutaient et se sollicitaient mutuellement. (Dossier nº 19)

Dans ce cas, le récit oral s'articule ensuite à une mise en commun de réactions, dans un échange intersubjectif productif.

Sont également proposés des jeux scéniques de textes narratifs ou des mises en scène de textes théâtraux. Ainsi une enseignante s'engage, pour étudier L'Amour médecin en classe de sixième, dans un projet didactique particulièrement fécond :

Comme Molière nous l'affirme dans son avis au lecteur : «les comédies ne sont faites que pour être jouées ". Je m’attaque donc à enseigner aux élèves comment, selon le souhait du dramaturge, "découvrir dans la lecture tout le jeu du théâtre", et comment s'initier au jeu théâtral.

Anne Ubersfeld rejoint cette analyse et explique dans Lire le théatre que «le texte de théâtre est troué» et que le lecteur est "contraint de boucher les trous du texte, autrement dit de se construire une représentation imaginaire». [...] Mon objectif didactique est de faire percevoir aux élèves le sens comique de l'œuvre [...] Lexpérience concrète et incarnée du jeu théâtral va leur permettre d'actualiser le sens du texte et d'éclairer la singularité des rapports entre les personnages. $\left(\right.$ Dossier $\left.n^{\circ} 49\right)$

Le projet d'ouvrir les élèves à la compréhension du texte par le jeu théâtral, d'engager par le jeu une implication voire une identification à un personnage nous semble être susceptible de favoriser une lecture riche et personnelle de l'œuvre. Cet objectif nous semble tout à fait proche de ce qu' envisage Anick Brillant-Annequin, à propos des pièces de théâtre : 
De nombreux exercices permettent de partir du jeu pour aller vers le texte, familiarisant ainsi le lecteur avec une structure globale des échanges, des personnages et des enjeux, ce qui facilite la lecture fine de la pièce et la compréhension d'une langue qui est perçue si souvent comme une langue étrange, voire étrangère ${ }^{18}$.

Tel est également ce que souligne l'enseignante, qui explique la difficulté première de ses élèves à lire silencieusement le texte ${ }^{19}$, difficulté levée dès qu'elle les engage à jouer, s'appuyant notamment sur les ressources non verbales ${ }^{20}$ que recèle la pièce, sur les costumes ${ }^{21}$, et même les lumières ou les musiques ${ }^{22}$. Là encore, elle paraît totalement s'inscrire dans les préconisations d'Anick Brillant-Annequin :

Il y a souvent une entrée possible dans un texte théâtral qui correspond aux aptitudes et goûts spécifiques de chaque lecteur. C'est l'occasion de valoriser la lecture sonore et musicale des musiciens ou amateurs de musiques, la lecture corporelle et spatiale des sportifs et danseurs, la lecture picturale ou architecturale des plasticiens et créateurs manuels, la lecture de la lumière des techniciens de l'électricité ainsi que la lecture des costumes, maquillages et coiffures qui intéressent de nombreux élèves ${ }^{23}$.

Cet exemple nous paraît particulièrement éloquent car il montre combien certaines activités orales, engagées pour favoriser une compréhension du texte, permettent à chaque élève de s'exprimer, de s'approprier personnellement le texte.

\section{Le rapprochement de la lecture scolaire avec les pratiques de lecture sociales ou privées}

De nombreuses propositions des enseignants peuvent enfin s'inscrire dans le projet d'un rapprochement entre les pratiques scolaires et les pratiques sociales

I8. A. Brillant-Annequin, "Lire des pièces de théâtre : le pari de l'impossible?», LSL, p. 267277, p. 276.

19. "La lecture personnelle de la pièce a été très difficile pour les élèves, seuls trois sur vingt-six l'ont vraiment appréciée et comprise et sont capables d'en parler. Tous les autres ont été freinés dans leur lecture par le vocabulaire et les constructions syntaxiques. Les notes de bas de page n'ont pas toujours suffi, une des élèves a même cru que Lisette était un animal à cause du mot «domestique». Je les rassure en leur rappelant l'assertion de Molière «les comédies ne sont faites que pour être jouées", nous allons donc étudier L'Amour médecin en tant que spectacle vivant" (extrait du dossier $\mathrm{n}^{\circ} 49$ ).

20. «La mise en abyme théâtrale fait apparaître les savoureux doubles sens des codes gestuels avec les effets de la double énonciation ", à propos de la scène où Clitandre met un anneau au doigt de Lucinde pour la guérir/l'épouser» (extrait du dossier n 49 ).

2I. "Ayant compris l'importance du costume, les élèves réfléchissent désormais à celui qu'ils pourraient choisir pour mieux incarner leur personnage» (extrait du dossier $n^{\circ} 49$ ).

22. "Ils choisissent parmi deux musiques aux tempos volontairement contrastés celle qui accompagnera leur prestation" (extrait du dossier $n^{\circ} 49$ ).

23. A. Brillant-Annequin, LSL, p. 277. 
ou artistiques de lecture. Dans cette perspective, plusieurs dispositifs visent à initier les élèves à tenir des rôles sociaux tels que ceux de jury, de membres de comité de lecture ou de libraires et de bibliothécaires. Ainsi, plusieurs professeurs engagent leur classe dans la participation à des sélections de prix littéraires, d'autres proposent des travaux en collaboration avec le CDI, les bibliothèques ou même des librairies : les élèves sont par exemple conduits, dans un dossier, à rédiger les bandeaux rouges qui seront placés autour des livres, à préparer des post-it collés devant les ouvrages présentés ou encore à glisser leur commentaire dans la revue de la bibliothèque ${ }^{24}$. Dans un autre dossier, l'enseignant a proposé aux élèves de commander eux-mêmes les ouvrages du CDI, ce qui les a conduit à rechercher, discuter, exprimer leur goût et leur préférence avant d'établir des listes de commande ${ }^{25}$.

Un autre type de pratique vise à favoriser la rencontre des lecteurs-élèves avec le monde des artistes, auteurs ou interprètes qui deviennent alors médiateurs de l'accès à l'œuvre, ou encore à permettre aux élèves d'assister à une représentation. La rencontre avec l'œuvre se trouve dès lors actualisée et facilitée par le spectacle, dans lequel le texte se donne. Ainsi, une enseignante ayant amené ses élèves de première à un spectacle sur Gargantua, explique que celui-ci «a permis aux élèves de s'approprier différemment l'héritage de la pensée humaniste renaissante, et de le rendre vivant à travers des souvenirs concrets». Être spectateur permet alors, en allégeant la tâche du lecteur, de développer plus facilement la subjectivité de la réception et ainsi de l'exercer. En outre, la rencontre avec un auteur ou un comédien amène également le lecteur à reconnaître un scripteur ou artiste subjectif, et à se sentir également reconnu comme sujet lecteur. C'est ce que manifeste cette enseignante qui rapporte cette anecdote issue de la rencontre avec Michèle Castelli, auteur d'Un chat qui vient de loin:

[Les élèves] ont fait à plusieurs reprises des comparaisons entre Alice et le Chat qui me semblaient pertinentes. Finalement, la réalisation du projet nous montrera que ce parallèle n'avait jamais été envisagé par l'auteur de façon consciente! $\left(\right.$ Dossier $\left.n^{\circ} 90\right)$

Grâce à la rencontre avec l'auteur, les élèves prennent conscience de la subjectivité de leur lecture puisque le lien intertextuel qu'ils ont perçu collectivement n'avait pas été conçu par l'auteur. Ils reconnaissent, comme le souligne Vincent Jouve, que «nous ne pouvons faire autrement que construire le sens en nous référant à un déjà lu forcément subjectif. [...] Lire, c'est faire jouer, sans 
souci de chronologie, toutes les connexions possibles entre les textes ${ }^{26}{ }^{\prime}$. De plus, l'auteur reconnaît la pertinence de leur lecture, sa richesse, et s'interroge à son tour sur sa démarche d'écriture : ainsi la rencontre devient celle d'une écriture subjective avec une lecture subjective, qui se trouve de surcroît valorisée.

Nous conclurons en présentant un dispositif qui nous semble davantage prendre en compte la lecture dans sa dimension intime et privée : il consiste à ménager, après la lecture orale du texte et avant l'étude proprement dite, un temps de silence, en proposant éventuellement de noter quelques mots évocateurs :

En affinant ma pratique autour de ce dispositif, j'ai pris conscience de l'importance à accorder aux transitions entre chacune de ses situations. Cet "espace-temps» de quelques minutes entre la lecture et l'interaction orale pendant lequel les élèves ont dû transcrire en quelques mots au crayon leurs émotions de lecteur est primordial. (Dossier $n^{\circ}$ 19)

Il semble bien que, dans ce cas, le professeur permette le développement d'une relation intime avec le texte et l'accueil des émotions qu'il suscite. À travers la délimitation d'un moment spécifique dans le temps de la séance, est alors reconnue la nécessité d'une rencontre intime avec ce que dit le texte, avec les échos qu'il déploie en chacun.

\section{Difficultés et limites d'un enseignement de la lecture subjective}

Ainsi, les dossiers analysés présentent de très nombreux dispositifs, souvent innovants et créatifs, qui permettent de développer des compétences de lecture subjective. Certaines expériences, fort riches, apportent même de nouvelles propositions pédagogiques. Cependant plusieurs nuances sont à apporter à cette analyse qualitative encourageante. En effet, bien que notre étude ait plutôt cherché à montrer la vitalité d'une réflexion sur les pratiques que la qualité des réalisations, l'étude des dossiers nous amène cependant à identifier plusieurs limites et difficultés de mise en ouvre, parfois pointées par les enseignants eux-mêmes, notamment dans leur analyse réflexive.

Tout d'abord il est nécessaire de mesurer l'importance effective que prennent les activités de lecture subjective dans l'ensemble de la séquence didactique présentée. Comme nous l'avons évoqué en introduction, peu de dossiers accordent plus d'une activité à une telle approche. Bien souvent, les enseignants se limitent à proposer des séances de lecture analytique, par ailleurs pertinentes, sans véritablement engager les élèves dans leur lecture. Quant aux pratiques

26. V. Jouve, $L S L$, p. Io8. 
de lecture subjective, elles se trouvent envisagées, le plus souvent, dans le cadre de conduite de projets pédagogiques de tous types - écriture, rencontre, production de spectacle. Ainsi, le temps de la prise en compte de l'élève sujet lecteur serait celui, exceptionnel, d'un projet, dont on sait qu'il ne va occuper qu'un temps réduit dans l'année. Il nous semble alors nécessaire d'amener les enseignants à dépasser l'idée que la pratique d'une lecture subjective doit être exceptionnelle, liée à un projet spécifique, et qu'elle ne peut pas devenir une posture coutumière durablement inscrite dans la classe.

Une autre difficulté semble résider dans l'articulation entre lecture littéraire et lecture subjective : en effet, les fonctions de l'écrit où se consignent les traces d'une lecture ne sont pas toujours véritablement comprises, et des confusions conduisent à trouver, dans ces supports, des activités relevant de la tradition scolaire, ce qui en fausse la portée et l'intérêt. Ceci conduit certains des "cahiers de lecteurs" à comprendre aussi bien des écrits subjectifs que des lectures plus distantes et formelles, conduites en classe ${ }^{27}$. De manière corélée, on note un flou dans les objectifs que les enseignants se fixent à travers la réalisation de ces écrits : ainsi, l'auteur de ce dossier, qui propose la composition d'un journal du lecteur, en présente les objectifs suivants : "Cette partie permettra de commencer l'activité du “journal du lecteur", visant à aider l'appropriation progressive d'une culture littéraire et aussi à inciter à un échange productif entre les élèves de la classe» (dossier $\mathrm{n}^{\circ} 56$ ). Pour cet enseignant, l'objectif du journal de lecteur consiste à permettre à l'élève de rendre compte de toutes les lectures abordées pendant l'année de façon à se les approprier dans la perspective de la constitution d'une culture commune. De même, le journal constitue un support pour l'échange oral, une étape vers d'autres activités et non une fin en soi. Même si ces perspectives sont intéressantes, elles peuvent dénaturer la raison d'être d'un journal de lecteur qui vise plutôt, en premier lieu, à constituer le recueil de la subjectivité du lecteur et le support d'une résonance de l'œuvre en lui.

De plus, plusieurs dossiers manifestent une difficulté des enseignants à corriger de tels travaux. Certains proposent des évaluations de lecture parallèles, d'autres expliquent qu'ils ont fait le choix d'évaluation formative - notamment pour des travaux valorisant la dimension esthétique des productions - conduisant à une écriture en plusieurs jets, d'autres encore décident de ne pas évaluer ces travaux. À nouveau ici, une difficulté de mise en œuvre se dessine, et aucun

27. Ainsi, une enseignante propose aux élèves, dans leur carnet de lecture, de compléter une biographie «à trous» de Lewis Carroll (dossier $\mathrm{n}^{\circ}$ 53), ou fait travailler sur la comparaison incipit et excipit en proposant une activité formelle : «je leur ai demandé dans un premier temps de lire silencieusement les deux textes, et j'ai noté au tableau ce qu'ils devaient ensuite chercher dans les textes : les lieux, les personnages, les temps verbaux et les étapes du récit dans l'incipit». 
dossier ne semble véritablement proposer une solution satisfaisante sur cette question. Ce problème se rencontre également dans les pratiques orales, où un flou sur l'objectif réel de l'enseignant - acquérir des compétences orales, lire en s'investissant, vérifier une compréhension - est perceptible et perturbe toute évaluation, et où l'investissement subjectif de l'élève semble n'avoir pas toujours été visé a priori.

Ajoutons, enfin, que dans le cadre de pratiques orales nouvelles, où les réactions de chacun sont accueillies, où le professeur ne détient plus la parole absolue, et où les sujets sont renvoyés à des questionnements personnels parfois profonds, la posture de l'enseignant doit évoluer : elle doit accompagner la parole plutôt que la diriger, rassurer des identités de lecteur en construction et accompagner des identités personnelles qui parfois s'ébranlent, et se reconfigurent. Plusieurs enseignants reconnaissent cette évolution de posture et soulignent qu'ils ont dû modifier leur pratique frontale vis-à-vis du groupe classe. Il est ainsi nécessaire que l'enseignant se révèle comme lecteur subjectif auprès de ses élèves, qu'il se représente dans une "lecture jouée», comme la nomme Patrick Demougin, dans laquelle il explore lui-même les différentes postures de lecteur. L'élève alors, guidé, rassuré de voir comment se lient une œuvre et un sujet, conduira lui-même ses réajustements, et pourra construire son propre rapport au texte. Bien entendu, cette proposition engage, en matière de formation des enseignants, de nouvelles approches, où l'enseignant pourrait être conduit à parler de ses lectures, des résonances d'une œuvre en lui, à ouvrir la porte de ce que Patrick Chamoiseau appelle sa sentimenthèque. 